\title{
Photoluminescence-based measurements of the energy gap and diffusion length of $\mathbf{Z n}_{3} \mathbf{P}_{2}$
}

\author{
Gregory M. Kimball, ${ }^{\text {a) }}$ Astrid M. Müller, Nathan S. Lewis, and Harry A. Atwater \\ Noyes Laboratory, Watson Laboratory and Beckman Institute, California Institute of Technology, Pasadena, \\ California 91125, USA
}

(Received 29 June 2009; accepted 20 August 2009; published online 14 September 2009)

\begin{abstract}
The steady-state photoluminescence spectra of zinc phosphide $\left(\mathrm{Zn}_{3} \mathrm{P}_{2}\right)$ wafers have revealed a fundamental indirect band gap at $1.38 \mathrm{eV}$, in close proximity to the direct band gap at $1.50 \mathrm{eV}$. These values are consistent with the values for the indirect and direct band gaps obtained from analysis of the complex dielectric function deduced from spectroscopic ellipsometric measurements. Bulk minority carrier lifetimes of $20 \mathrm{~ns}$ were observed by time-resolved photoluminescence decay measurements, implying minority-carrier diffusion lengths of $\geq 7 \mu \mathrm{m}$. () 2009 American Institute of Physics. [doi:10.1063/1.3225151]
\end{abstract}

Zinc phosphide $\left(\mathrm{Zn}_{3} \mathrm{P}_{2}\right)$ has significant potential as an absorber in thin film photovoltaics. $\mathrm{Zn}_{3} \mathrm{P}_{2}$ has been reported to have a direct, $1.5 \mathrm{eV}$ band gap, high $\left(>10^{4}-10^{5} \mathrm{~cm}^{-1}\right)$ light absorbance in the visible region, ${ }^{1}$ and long $(5-10 \mu \mathrm{m})$ minority-carrier diffusion lengths. ${ }^{2}$ To date, $\mathrm{Zn}_{3} \mathrm{P}_{2}$ has been produced almost exclusively with $p$-type doping, ${ }^{3}$ preventing the fabrication of $p-n$ homojunctions. Solar cells using $\mathrm{Zn}_{3} \mathrm{P}_{2}$ have therefore been constructed from Schottky contacts, $p-n$ semiconductor heterojunctions, ${ }^{4}$ or liquid contacts, ${ }^{5}$ with $p-\mathrm{Zn}_{3} \mathrm{P}_{2} / \mathrm{Mg}$ Schottky diodes having exhibited $>6 \%$ solar energy-conversion efficiency. ${ }^{6}$

However, even the basic materials parameters of $\mathrm{Zn}_{3} \mathrm{P}_{2}$, such as the energy gap, remain controversial. A direct interband transition has been observed at $\sim 1.5 \mathrm{eV}$, but the more gradual decline in absorption between 1.3-1.5 eV indicates that either an indirect gap and/or a defect band contributes to the absorption tail. ${ }^{7-12}$ Some absorption measurements have led to assignments of the fundamental band gap as direct, ${ }^{7-9}$ whereas others have concluded that the fundamental gap is indirect. $^{10-12}$ Computational methods using empirical pseudopotentials have also yielded both direct ${ }^{13-15}$ and indirect ${ }^{13,16}$ transitions at the fundamental band gap, depending whether the crystal structure is modeled as a cubic antifluorite or as a tetragonal structure. A fundamental indirect gap is supported by investigations of the spectral response properties of $\mathrm{Mg}$ Schottky diodes ${ }^{17}$ and of aqueous photoelectrochemical cells, ${ }^{5}$ which have produced estimates of an indirect gap at 1.37 and $1.43 \mathrm{eV}$, respectively.

To better elucidate the band structure of $\mathrm{Zn}_{3} \mathrm{P}_{2}$, we have performed steady-state photoluminescence (PL) measurements of $\mathrm{Zn}_{3} \mathrm{P}_{2}$ near room temperature. In addition, we report spectroscopic ellipsometric data on $\mathrm{Zn}_{3} \mathrm{P}_{2}$ and the $n(\omega)$, $k(\omega)$ spectra derived therefrom. Finally, we have performed time-resolved PL decays on bulk crystalline $\mathrm{Zn}_{3} \mathrm{P}_{2}$, to explore the minority-carrier lifetimes of this material.

The $\mathrm{Zn}_{3} \mathrm{P}_{2}$ samples used in this study were grown by physical vapor transport. Red phosphorus chips and zinc shot (99.9999\%, Alfa Aesar) were combined at $850{ }^{\circ} \mathrm{C}$ to form $\mathrm{Zn}_{3} \mathrm{P}_{2}$ powders. Using procedures described previously, ${ }^{18-23}$ the powders were then grown into polycrystalline boules that

${ }^{\text {a)} E l e c t r o n i c ~ m a i l: ~ g r e g o r y k @ c a l t e c h . e d u . ~}$ were $1 \mathrm{~cm}$ in diameter and $4 \mathrm{~cm}$ in length, with grain sizes of $\sim 1-5 \mathrm{~mm}^{2}$ and hole concentrations of $10^{15} \mathrm{~cm}^{-3}$. The resulting crystals were diced with a diamond saw and were polished with diamond paste to produce $\mathrm{Zn}_{3} \mathrm{P}_{2}$ wafers with a 1-2 $\mathrm{nm}$ root-mean-squared surface roughness, as measured by atomic force microscopy. Samples with $1 \mathrm{~cm}$ diameter and $\sim 700 \mu \mathrm{m}$ thickness were etched for $30 \mathrm{~s}$ in $2 \%-3 \%$ (v:v) $\mathrm{Br}_{2}$ in $\mathrm{CH}_{3} \mathrm{OH}$, rinsed in $\mathrm{CH}_{3} \mathrm{OH}$, dried under a stream of $\mathrm{N}_{2}$, and used promptly thereafter. No contamination by oxides of zinc or phosphorus, but $\sim 2 \mathrm{ML}$ of elemental $\mathrm{P}$, was observed through $\mathrm{X}$-ray photoelectron spectroscopy on $\mathrm{Br}_{2}$-etched $\mathrm{Zn}_{3} \mathrm{P}_{2}$ samples.

PL measurements were collected using a closed-cycle cryostat pumped to $5 \times 10^{-6}$ Torr. For steady-state PL measurements, excitation was performed using the $488 \mathrm{~nm}$ line of an Ar-ion laser that was chopped at $10 \mathrm{kHz}$ using an acousto-optic modulator. The emission was passed through a monochromator and focused onto an infrared photomultiplier tube, and the PL signal was monitored using a lock-in amplifier. Time-resolved PL measurements were performed at $10 \mathrm{kHz}$ with $70 \mathrm{ps}, 355 \mathrm{~nm}$ pulses produced by frequencytripling of regeneratively amplified, mode-locked Nd:YAG (yttrium aluminum garnet) laser pulses. The emission was collected using a fiber optic and the signal was integrated using a streak camera. Spectroscopic ellipsometry was performed at an angle of incidence of $50^{\circ}, 60^{\circ}$, and $70^{\circ}$, respectively, for $300 \mathrm{~nm}<\lambda<2200 \mathrm{~nm}$ with a Xe lamp visible light source and a Fourier-transform infrared spectrometer. The $\psi(\omega), \Delta(\omega)$ data were converted to $n(\omega), k(\omega)$ values assuming a bulk, isotropic substrate.

Between 5 and $20 \mathrm{~K}, \mathrm{Zn}_{3} \mathrm{P}_{2}$ samples exhibited an intense PL signal at $1.36 \mathrm{eV}$, as well as a phonon replica at $1.32 \mathrm{eV}$ (Fig. 1, top panel). The $43 \mathrm{meV}$ energy of the phonon is in agreement with the longitudinal optical phonon observed in previous low-temperature PL studies of $\mathrm{Zn}_{3} \mathrm{P}_{2} \cdot{ }^{24}$ Between 200 and $310 \mathrm{~K}$, steady-state PL spectra showed a peak near $1.4 \mathrm{eV}$, along with a shoulder at $\sim 1.5 \mathrm{eV}$ that became more prominent at higher temperatures (Fig. 1, lower panel). In addition to the two main peaks, a smaller feature at $1.37 \mathrm{eV}$ that saturated at higher excitation intensity, was observed, as would be expected for defect luminescence. A small feature at $1.30 \mathrm{eV}$ was produced by a diffracted laser line. 


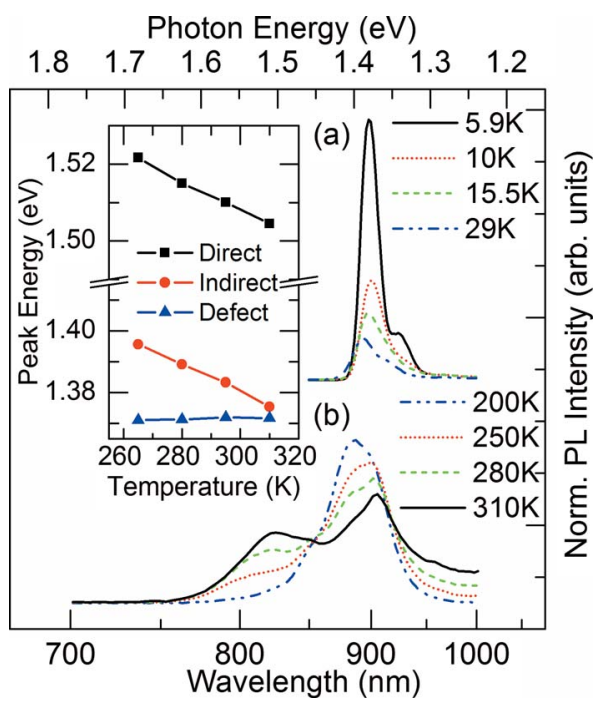

FIG. 1. (Color online) Normalized PL spectra of $\mathrm{Br}_{2}$-etched $\mathrm{Zn}_{3} \mathrm{P}_{2}$ wafers in the temperature ranges (a) 5.9-29 K and (b) 200-310 K. The inset shows the energies, as a function of temperature, of the direct and indirect interband transitions, as well as a defect-assisted transition.

Fitting of the data obtained at a range of laser excitation intensities at each temperature yielded precise positions of the centers of the PL peaks. At room temperature, the two main peaks were centered at $1.38 \pm 0.01$ and $1.50 \pm 0.01 \mathrm{eV}$ (Fig. 1, inset). With decreasing temperature, the peaks centered at 1.38 and $1.50 \mathrm{eV}$ exhibited (blue)shifts of -3.8 $\times 10^{-4}$ and $-4.5 \times 10^{-4} \mathrm{eV} / \mathrm{K}$, respectively, broadly consistent with the well-documented energy gap changes of III-V compound semiconductors. ${ }^{25}$

As observed previously, ${ }^{24}$ at cryogenic temperatures, the PL signal exhibited a significant blueshift as the laser excitation intensity was increased. In contrast, near room temperature, the centers of the peaks at 1.38 and $1.50 \mathrm{eV}$ did not change with increasing pump power (Fig. 2). The lack of a shift in the centers of 1.38 and $1.50 \mathrm{eV}$ peaks in response to increasing pump power suggests a high density of states in the $\mathrm{Zn}_{3} \mathrm{P}_{2}$ and is consistent with these signals being derived from interband transitions. Hence, at room temperature, the steady-state PL spectra can be ascribed to luminescence from both the fundamental indirect gap at $1.38 \mathrm{eV}$ and the nearby

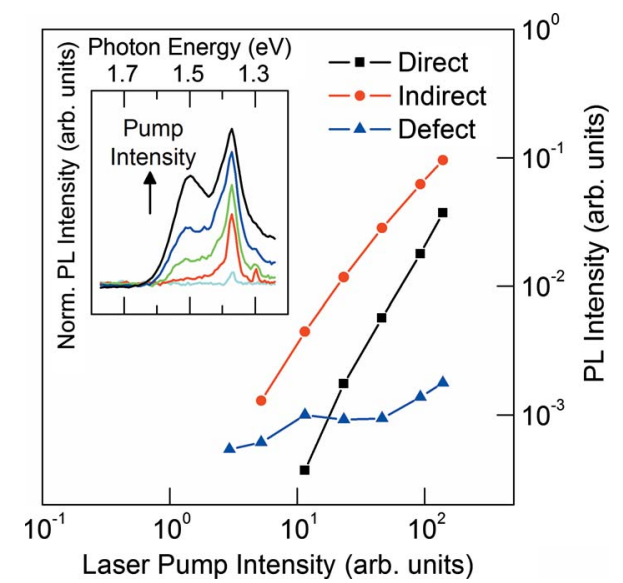

FIG. 2. (Color online) Relative intensities of the direct gap, indirect gap, and defect-assisted $\mathrm{PL}$ of $\mathrm{Br}_{2}$-etched $\mathrm{Zn}_{3} \mathrm{P}_{2}$ wafers as a function of laser pump intensity at $295 \mathrm{~K}$. The inset shows the normalized PL spectra across the same range of laser pump intensities.

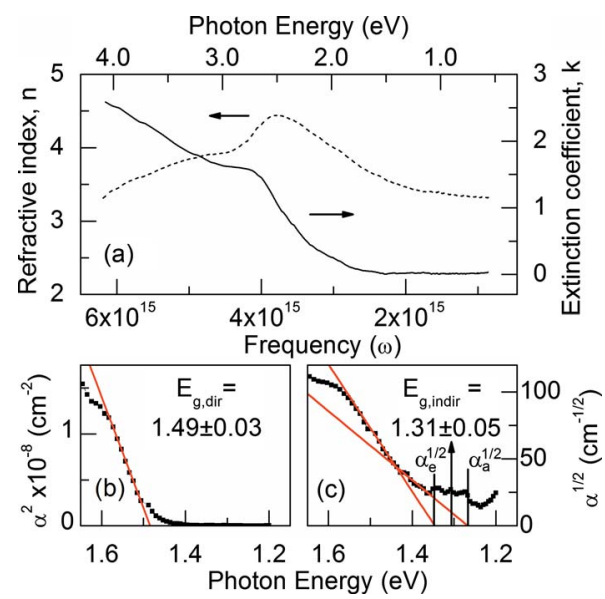

FIG. 3. (Color online) Spectroscopy ellipsometric analysis of $\mathrm{Br}_{2}$-etched $\mathrm{Zn}_{3} \mathrm{P}_{2}$ wafers at $295 \mathrm{~K}$ showing: (a) the derived $n(\omega), k(\omega)$ data in the spectral range $0.5-4.0 \mathrm{eV}$; (b) the test of $\alpha(\omega)$ for direct transitions; and (c) the test of $\alpha(\omega)$ for indirect transitions.

direct gap at $1.50 \mathrm{eV}$. Similar direct and indirect gap PL features have been reported for room temperature radiative recombination in germanium, ${ }^{26}$ which is a material with a fundamental indirect transition at $0.66 \mathrm{eV}$ and a direct interband transition at $0.80 \mathrm{eV}$.

The $\psi(\omega), \Delta(\omega)$ (amplitude ratio and phase shift, respectively, of the reflectance at frequency $\omega$ ) behavior obtained from spectroscopic ellipsometric measurements on bulk $\mathrm{Zn}_{3} \mathrm{P}_{2}$ samples was used to generate values for the refractive index $n(\omega)$ and extinction coefficient, $k(\omega)$, functions of $\mathrm{Zn}_{3} \mathrm{P}_{2}$. Figure 3(a) shows representative $n(\omega), k(\omega)$ data for $\mathrm{Zn}_{3} \mathrm{P}_{2}$ polycrystalline wafers over the $0.5-4.1 \mathrm{eV}$ spectral range. Inclusion of a 1-2 nm overlayer in the model, to account for surface roughness and chemical overlayers, was found to be of second order to the derived $n(\omega), k(\omega)$ data. The derived spectral data for the absorption coefficient function, $\alpha(\omega)$, for $\mathrm{Zn}_{3} \mathrm{P}_{2}$ indicated a direct transition at $1.49 \pm 0.03 \mathrm{eV}$ [Fig. 3(b)] and an indirect transition at $1.31 \pm 0.05 \mathrm{eV}$ [Fig. 3(c)]. The $\alpha^{1 / 2}$ plots versus energy shows linear regions associated with both phonon absorption $\alpha_{a}^{1 / 2}$ and emission $\alpha_{e}^{1 / 2}$ yielding $E_{g}+E_{p}$ and $E_{g}-E_{p}$ intercepts that indicated $E_{g}=1.31 \pm 0.05 \mathrm{eV}$ and $E_{p}=0.04 \pm 0.02 \mathrm{eV}$. The linearity of the $\alpha^{2}$ and $\alpha^{1 / 2}$ plots versus energy is consistent with the presence of both direct and indirect gaps, but the resolution for extinction measurements at low values of $k$ $(<0.07)$ made the values of the band gap energies determined by spectroscopic ellipsometry less precise than those derived from the PL data. The $n(\omega), k(\omega)$ data indicated that $\mathrm{Zn}_{3} \mathrm{P}_{2}$ has a very high optical absorption, with $\alpha$ $>10^{4} \mathrm{~cm}^{-1}$ throughout the visible region. The feature in the $n(\omega), k(\omega)$ data at $2.5 \mathrm{eV}$ indicates a high density of states, presumably associated with a higher lying band, perhaps the at $\Gamma_{25}$ point. ${ }^{14}$ This $n(\omega), k(\omega)$ data set, that extends through the entire visible spectrum, is expected to be valuable for future optoelectronic device modeling of $\mathrm{Zn}_{3} \mathrm{P}_{2}$ solar cells.

Time-resolved PL measurements at room temperature were well fitted by a double exponential decay, with time constants of $4 \mathrm{~ns}$ and $20 \mathrm{~ns}$ describing the decay behavior for excitation levels of $0.5-10 \mathrm{~nJ} / \mathrm{pulse}$ (Fig. 4). The doubleexponential behavior suggests that these time constants are related to both bulk and surface recombination processes. Minimal surface potentials have been reported for $\mathrm{Br}_{2}$-etched 


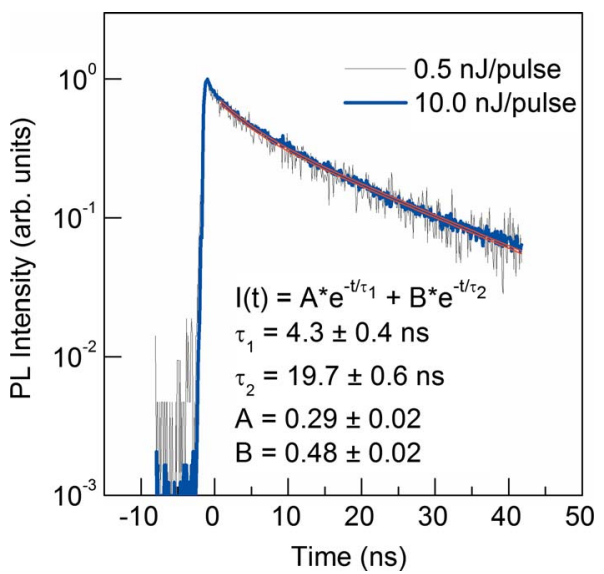

FIG. 4. (Color online) Time-resolved PL decay measurements of $\mathrm{Br}_{2}$-etched $\mathrm{Zn}_{3} \mathrm{P}_{2}$ wafers at $295 \mathrm{~K}$, showing double-exponential decay behavior.

$\mathrm{Zn}_{3} \mathrm{P}_{2}{ }^{27}$ However, the $\mathrm{Zn}_{3} \mathrm{P}_{2}$ wafer very strongly absorbs the $355 \mathrm{~nm}$ pump light, with a $90 \%$ absorbance depth of $\sim 8 \mathrm{~nm}$. With a high concentration of photogenerated carriers near the surface, it seems reasonable to suggest that the observed PL decay rates are thus dominated by surface recombination. Hence, assuming an electron mobility of $1000 \mathrm{~cm}^{2} \mathrm{~V}^{-1} \mathrm{~s}^{-1},{ }^{17}$ the minority-carrier recombination lifetimes measured in our work would correspond to minoritycarrier diffusion lengths of $\geq 7 \mu \mathrm{m}$, which is promising for the use of such $\mathrm{Zn}_{3} \mathrm{P}_{2}$ materials as a solar cell absorber.

This work was supported by the Office of Energy Efficiency and Renewable Energy, U.S. Department of Energy under Grant No. DE-FG36-08GO18006, as well as Caltech Center for Sustainable Energy Research (CCSER). We acknowledge use of facilities supported by the Beckman Institute Laser Resource Center (BILRC). One of us (G.M.K.) acknowledges support under an NDSEG graduate fellowship.

${ }^{1}$ E. A. Fagen, J. Appl. Phys. 50, 6505 (1979).

${ }^{2}$ N. C. Wyeth and A. Catalano, J. Appl. Phys. 50, 1403 (1979).

${ }^{3}$ A. Catalano and R. B. Hall, J. Phys. Chem. Solids 41, 635 (1980).

${ }^{4}$ F. C. Wang, A. L. Fahrenbruch, and R. H. Bube, J. Appl. Phys. 53, 8874 (1982).

${ }^{5}$ M. Bhushan, J. A. Turner, and B. A. Parkinson, J. Electrochem. Soc. 133, 536 (1986).

${ }^{6}$ M. Bhushan and A. Catalano, Appl. Phys. Lett. 38, 39 (1981).

${ }^{7}$ J. Misiewicz, J. Phys.: Condens. Matter 2, 2053 (1990).

${ }^{8}$ A. Nayak, D. R. Rao, and H. D. Banerjee, Solid State Commun. 78, 149 (1991).

${ }^{9}$ J. M. Pawlikowski, Phys. Rev. B 26, 4711 (1982).

${ }^{10}$ V. Munoz, J. Appl. Phys. 60, 3282 (1986).

${ }^{11}$ J. M. Pawlikowski, J. Misiewicz, and N. Mirowska, J. Phys. Chem. Solids 40, 1027 (1979).

${ }^{12}$ L. Zdanowicz, Acta Phys. Pol. A 57, 159 (1980).

${ }^{13}$ J. Andrzejewski and J. Misiewicz, Phys. Status Solidi B 227, 515 (2001)

${ }^{14}$ P. J. Linchung, Phys. Status Solidi B 47, 33 (1971).

${ }^{15}$ J. Misiewicz and J. Andrzejewski, Phys. Status Solidi B 184, K7 (1994).

${ }^{16}$ K. Sieranski, Phys. Rev. B 50, 7331 (1994).

${ }^{17}$ M. Bhushan, J. Appl. Phys. 53, 514 (1982).

${ }^{18}$ A. Catalano, J. Cryst. Growth 49, 681 (1980).

${ }^{19}$ S. Fuke, J. Cryst. Growth 87, 567 (1988).

${ }^{20}$ A. Kuroyanagi, J. Cryst. Growth 100, 1 (1990).

${ }^{21}$ J. Misiewicz, F. Krolicki, M. Lewicki, and J. F. Kasprzak, Acta Phys. Pol. A 69, 1127 (1986).

${ }^{22}$ F.-C. Wang, R. H. Bube, R. S. Feigelson, and R. K. Route, J. Cryst. Growth 55, 268 (1981).

${ }^{23}$ G. M. Kimball, Proceedings of the 33rd IEEE Photovoltaic Specialists Conference, San Diego, CA, 2008 (unpublished).

${ }^{24}$ F. Briones, F. C. Wang, and R. H. Bube, Appl. Phys. Lett. 39, 805 (1981)

${ }^{25}$ Handbook Series on Semiconductor Parameters, edited by M. Levinshtein, S. Rumyantsev, and M. Shur (World Scientific, Singapore, 1996), Vol. 1.

${ }^{26}$ J. Haynes, Phys. Rev. 98, 1866 (1955).

${ }^{27}$ M. Casey, J. Appl. Phys. 61, 2941 (1987). 